\title{
HYDATID CYST IN A HEART FAILURE PATIENT MIMICKING PHANTOM TUMOR
}

\author{
Irmak İrem Özyiğit $^{1}$ (D), Beliz Koçyiğit ${ }^{1}$ (D), Servet Altay $^{2}$ (D)
}

${ }^{1}$ Trakya University School of Medicine, Edirne, TURKEY

${ }^{2}$ Department of Cardiology, Trakya University School of Medicine, Edirne, TURKEY

\begin{abstract}
Aims: We aimed to present a heart failure patient with a hydatid cyst of the lung, mimicking a phantom tumor. Case Report: A 71-year-old male patient presented to the cardiology department of Trakya University School of Medicine with increasing shortness of breath and cough. His hospital admission complaints, heart failure history, and chest radiology results were consistent with a phantom tumor of the lung secondary to congestive heart failure. The patient was given diuretics; however, there was no evidence of resolution or change in the size of the observed cystic lesion. A hydatid cyst as a differential diagnosis was considered, and subsequent questioning of the patient revealed animal contacts. The patient was offered the option of surgical removal of the cyst, which he declined due to the high mortality risk. The patient was prescribed $400 \mathrm{mg}$ of albendazole and was advised to have regular check-ups. In the long term, he showed no further signs and symptoms of hydatid cyst. The hydatid cyst was neither removed nor disappeared, and it continued to be visible on radiological follow-up examinations. Conclusion: Due to the similarities present in admission complaints and chest examinations, it is challenging to differentiate hydatid cyst of the lung in heart failure patients. Physicians should be aware of the hydatid cyst in the differential diagnosis of pleural cysts and consider patients' occupation and residency in order to not overlook zoonotic diseases. Keywords: Hydatid cyst, phantom tumor, congestive heart failure
\end{abstract}

\section{INTRODUCTION}

Human echinococcosis is a zoonosis caused by tapeworms of the genus Echinococcus (1). Echinococcus granulosus is responsible for cystic echinococcosis, also known as hydatidosis or hydatid disease $(1,2)$. Its definitive hosts are dogs and other carnivores such as red foxes, coyotes, and dingos (2). Its intermediate hosts are mainly sheep, cattle, goats, and pigs (2). The parasite is transmitted to definitive hosts when they consume the internal organs of intermediate hosts that contain the hydatid cysts $(1,2)$. Humans can be accidental hosts when the parasite is transmitted to them through the consumption of soil, water, or food that has come into contact with the feces of a definitive host (3).

Infestation with Echinococcus granulosus results in the formation of one or more hydatid cysts located primarily in the liver and lungs (2). The incubation period can last from a few months to many years (1). Most cases remain asymptomatic until the hydatid cysts become large enough for the patient to present with clinical symptoms (4). Clinical manifestations depend on the location of the cyst and may vary depending on its size (1). The most common symptoms of pulmonary cystic echinococcosis are cough, chest pain, dyspnea, and hemoptysis (5). Diagnosis of cystic echinococcosis is primarily by ultrasonography, supplemented by computed tomography and magnetic resonance imaging (4). Cysts may also be detected incidentally by imaging studies such as radiographs performed for other indications (4). In small, uncomplicated pulmonary cysts, benzimidazoles showed good efficacy when used alone (6). Preoperative administration of benzimidazoles should be avoided for larger lung cysts (6). Surgical interventions aimed at eliminating the parasite and treating the related pathology should be as conservative as possible (6). However, radical surgery may be required in cases of extensive parenchymal involvement, severe pulmonary suppuration, and complications (6).

When it comes to the differential diagnosis of hydatid cysts on chest X-ray, they can mimic various lung pathologies such as metastases, carcinoma, fluid-filled cysts, and inflammatory masses (7). In our case, the differential diagnosis was made with a phantom tumor. A phantom tumor is defined as an accumulation of fluid in the interlobar spaces of the lung, radiologically simulating a neoplasm, and usually secondary to congestive heart failure $(8,9)$. In this case report, we aim to present the diagnosis of a hydatid cyst mimicking a phantom tumor in a patient with heart failure.

\section{CASE REPORT}

A 71-year-old male patient was admitted to the cardiology department of Trakya University School of Medicine with increased shortness of breath and cough. He was an ex-smoker with a history of coronary artery disease (stents were implanted three years ago), hypertension, diabetes mellitus, myasthenia gravis, chronic obstructive pulmonary disease, congestive heart failure, ischemic cardiomyopathy, and atrial fibrillation. In the last three years, he frequently visited the emergency room with complaints of heart failure. A year ago, he was hospitalized for cardiopulmonary arrest where he received an implantable cardioverter-defibrillator for secondary protection. To treat his congestive heart failure and 
hypertension, he was treated with diuretics. He was also taking 2.5 $\mathrm{mg}$ of ramipril, sacubitril/valsartan, $15 \mathrm{mg}$ of rivaroxaban, $20 \mathrm{mg}$ of isosorbide dinitrate, $20 \mathrm{mg}$ of furosemide, and eplerenone.

The patient was admitted to the cardiology department for shortness of breath associated with his known congestive heart failure. The patient's X-ray revealed a cystic lesion in the lower side of the right lung (Figure 1). There was no evidence of a cystic lesion on the chest X-ray performed two months ago. Based on the history and radiological findings, he was diagnosed with a phantom tumor of the lung secondary to congestive heart failure. The patient received intensive diuretic treatment.

At follow-up, the physicians requested a contrast-enhanced computed tomography scan, which showed a cystic nodule with a smooth contour and a diameter of approximately $4 \mathrm{~cm}$ in the middle-lower lobes of the right lung (Figure 2). There was no evidence of resolution or change in the size of the observed cystic lesion. Although the location of the lesion and the history of heart failure suggested a diagnosis of phantom tumor, the lack of response to diuretic treatment ruled out this initial diagnosis. The patient was then consulted by the department of thoracic surgery who evaluated his radiological findings and medical history. They considered a hydatid cyst as a differential diagnosis, and subsequent questioning of the patient revealed that animal contacts and village housing supported this new diagnosis.

Following the diagnosis of a hydatid cyst, the patient was offered the option of surgical removal of the cyst, which he declined due to the high risk of the procedure. Subsequently, the patient was prescribed $400 \mathrm{mg}$ of albendazole and was advised to have regular check-ups. In the long term, he showed no further signs and symptoms of hydatid cyst. The hydatid cyst was neither removed nor disappeared, and it continued to be visible on radiological follow-up examinations (Figure 3). His hospitalizations related to heart failure continued, and the patient died in the cardiology department one year after the diagnosis of his hydatid cyst.

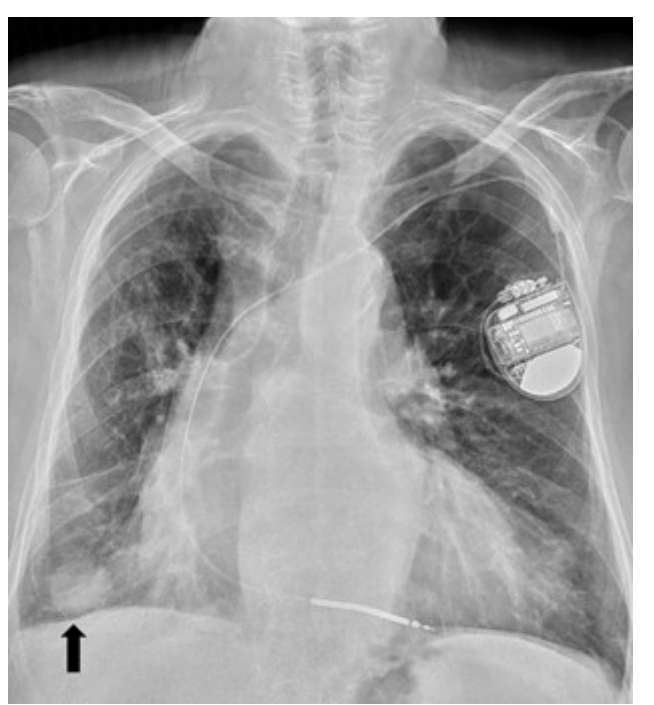

Figure 1: Posteroanterior chest radiography revealing hydatid cyst (Arrow).

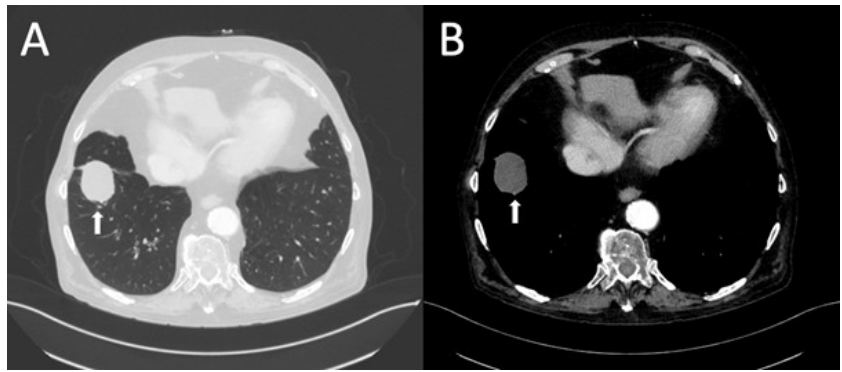

Figure 2: The computed tomography images reveal a hydatid cyst (Arrows). A: Lung parenchyma window. B: Mediastinal window.

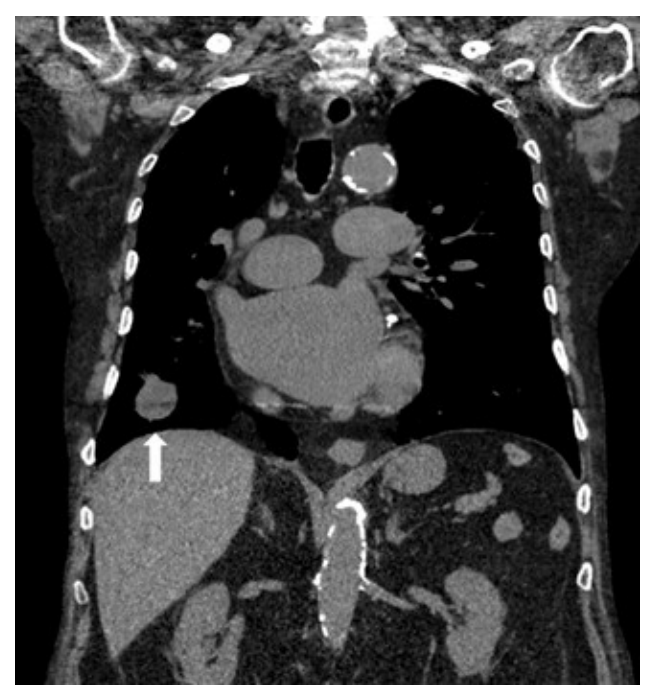

Figure 3: Coronal plane imaging of the patient. The computed tomography scan of the thorax shows a hydatid cyst (Arrow).

\section{DISCUSSION}

Hydatid cyst is the result of zoonotic infestation by tapeworms (1). The two main species of these tapeworms found in the alveolar system are Echinococcus granulosus and Echinococcus multilocularis (6). In our case, we are concerned with Echinococcus granulosus because this tapeworm is responsible for cystic echinococcosis, which we refer to as a "hydatid cyst" because of the disease it causes (1, 6). Accidental hosts, in this case humans, can get these parasites through contaminated products such as food or water that have come into contact with feces (3).

Patients with congestive heart failure tend to develop phantom tumors, and the right hemithorax is the most common area in the lung where they occur (10). Our patient's X-ray revealed a cystic mass on the lower side of the right lung, and he had a known history of heart failure. A presumptive diagnosis of a phantom tumor secondary to congestive heart failure was made. The rapid development of this mass supported this initial diagnosis. In patients with heart failure, it is advisable to think of a phantom tumor when a cystic mass appears on the X-ray to avoid unnecessary interventions and treatments (11).

The admission complaints of phantom tumors may include dyspnea and dry cough, which are very similar to symptoms of a hydatid cyst. Frequently encountered complaints include chest pain, cough, dyspnea, and hemoptysis in pulmonary cystic echinococcosis $(5,12)$. In our case, the patient presented with shortness of 
breath and cough, which in conjunction with his history was reminiscent of a phantom tumor. Similarly, in the case of Canpolat et al. (13), a patient presented with dyspnea and cough.

Phantom tumors are treatable with diuretics and a reduction in fluid intake (14). If the treatment plan is followed, complete regression of the tumor can be expected. In this case, the patient was treated with diuretics based on his initial diagnosis. Although the history of heart failure and location of the mass were relevant to phantom tumors, no regression or change in size was observed. Failure to respond to treatment was the main reason physicians considered other diagnoses. The final diagnosis was made based on chest X-rays and a history of animal contact. As a result, hydatid cyst was chosen as a differential diagnosis, which is endemic in our country (15). Demirci et al. (15) collected cases of hydatid cysts in a Turkish city and found that the lung was the second most common location. Definitive treatment of a hydatid cyst requires removal of the cyst by open surgery (16). In addition, albendazole can be added to the treatment plan preoperatively and/or postoperatively to prevent recurrence and achieve complete healing (16).

Complete healing was possible with surgical intervention and could prevent the compression caused by the mass, but our patient refused surgical removal of the cyst due to the high risk of mortality $(13,16)$. For this reason, only drug treatment, albendazole 400 $\mathrm{mg}$, was initiated. In the long term, the hydatid cyst was neither removed nor disappeared, and it was still visible on radiological follow-up examinations. Although the main drug treatment for hydatid cysts in the lungs, heart, and liver was albendazole, the study by Dehkordi et al. (16) showed that albendazole had no effect on cysts in the lungs.

The diagnosis of a hydatid cyst can be difficult in patients with heart failure because of the similarities between the symptoms on admission and chest X-rays. Physicians should think of hydatid cyst in the differential diagnosis of pleural lesions. Also, we recommend physicians consider the occupation and residence of their patients, especially to remind them of zoonotic diseases.

\section{Ethics Committee Approval: N/A}

Informed Consent: Informed verbal consent was obtained from the next of kin.

Conflict of Interest: The authors declare no conflict of interest.

Author Contributions: Concept: IİÖ, BK, SA. Design: IİÖ, BK, SA. Supervision: İIÖ, BK, SA. Resources: İÖ, BK, SA. Materials: IİÖ, BK, SA. Data collection and/or Processing: IİÖ, BK, SA. Analysis and/or Interpretation: IİO, BK, SA. Literature Search: İ̇Ö, BK, SA. Writing Manuscript: IİÖ, BK, SA. Critical Review: IIIÖ, BK, SA.
Financial Disclosure: The authors declared that this study received no financial support.

Acknowledgments: We would like to thank Fethi Emre Ustabaşığlu from the Radiology Department of Trakya University School of Medicine for their valuable guidance and contributions.

Editor-in-Chief's Note: Two of the authors of this article, Irmak İrem Özyiğit and Beliz Koçyiğit are members of the editorial board of Turkish Medical Student Journal. However, they did not take place at any stage of the editorial decision of the manuscript. The editors who evaluated this manuscript are from other institutions.

\section{REFERENCES}

1. McManus DP, Zhang W, Li J et al. Echinococcosis. Lancet 2003;362(9392):1295304.

2. Eckert J, Deplazes P. Biological, epidemiological, and clinical aspects of echinococcosis, a zoonosis of increasing concern. Clin Microbiol Rev 2004;17(1):107-35.

3. Alam MT, Saber S, Alam RF et al. Primary pulmonary hydatid disease. Bangladesh Crit Care J 2018;6(2):105-7.

4. Agudelo Higuita NI, Brunetti E, McCloskey C. Cystic echinococcosis. J Clin Microbiol 2016;54(3):518-23.

5. Santivanez S, Garcia HH. Pulmonary cystic echinococcosis. Curr Opin Pulm Med 2010;16(3):257-61.

6. Brunetti E, Kern P, Vuitton DA et al. Expert consensus for the diagnosis and treatment of cystic and alveolar echinococcosis in humans. Acta Trop 2010;114(1):116.

7. Garg MK, Sharma M, Gulati A et al. Imaging in pulmonary hydatid cysts. World J Radiol 2016;8(6):581-7.

8. Haus BM, Stark P, Shofer SL et al. Massive pulmonary pseudotumor. Chest 2003;124(2):758-60.

9. Sandal R, Jandial A, Mishra K et al. Phantom tumour and heart failure. BMJ Case Rep 2018;2018:bcr2018227364.

10. Gielen WP, Bijl M, Kofflard MJM. A phantom lung tumour in congestive heart failure. Neth Heart J 2006;14(10):345-6.

11. Argan O, Ural D. Phantom tumor of the lung in heart failure patient. Turk J Emerg Med 2017;17(3):121-2.

12. Kılıç D, Fındıkçığlu A, Bilen A et al. Management of complicated hydatid cyst of the thorax. ANZ J Surg 2007;77(9):752-7.

13. Canpolat U, Sunman H, Taher A et al. Fissural hydatid cyst in a young woman mimicking phantom tumor. Int J Cardiol 2010;140:61.

14. Lozo M, Lozo Vukovac E, Ivancevic Z et al. Phantom tumor of the lung: localized interlobar effusion in congestive heart failure. Case Rep Cardiol 2014;2014:207294.

15. Demirci E, Altun E, Çalık M et al. Hydatid cyst cases with different localization: region of Erzurum. Turkiye Parazitol Derg 2015;39(2):103-7.

16. Dehkordi AB, Sanei B, Yousefi M et al. Albendazole and treatment of hydatid cyst: review of the literature. Infect Disord Drug Targets 2019;19(2):101-4. 